\title{
Comparison of two numerical algorithms for computing the effects of mistuning of fan flutter
}

\author{
L. Salles \\ Imperial College London \\ Mechanical Engineering Department \\ Exhibition Road, \\ London SW7 2AZ, UK \\ I.salles@imperial.ac.uk
}

\author{
M. Vahdati \\ Imperial College London \\ Mechanical Engineering Department \\ Exhibition Road, \\ London SW7 2AZ, UK \\ m.vahdati@imperial.ac.uk
}

\section{ABSTRACT}

The aim of this paper is to study the effects of mistuning on fan flutter and to compare the prediction of two numerical models of different fidelity. The high fidelity model used here is a three-dimensional, whole assembly, time-accurate, viscous, finite-volume compressible flow solver. The Code used for this purpose is AU3D, written in Imperial College and validated for flutter computations over many years. To the best knowledge of authors, this is the first time such computations have been attempted. This is due to the fact that, such non-linear aeroelastic computations with mistuning require large amount of CPU time and cannot be performed routinely and consequently, faster (low fidelity) models are required for this task. Therefore, the second model used here is the aeroelastic fundamental mistuning model (FMM) and it based on an eigenvalue analysis of the linearized modal aeroelastic system with the aerodynamic matrix calculated from the aerodynamic influence coefficients. The influence coefficients required for this algorithm are obtained from the time domain non-linear Code by shaking one blade in the datum (tuned) frequency and mode. Once the influence coefficients have been obtained, the computations of aero damping require minimal amount of CPU time and many different mistuning patterns can be studied. The objectives of this work are to:

1. Compare the results between the two models and establish the capabilities/limitations of aeroelastic FMM

2. Check if the introduction of mistuning would bring the experimental and computed flutter boundaries closer,

3. Establish a relationship between mistuning and damping.

A rig wide-chord fan blade, typical of modern civil designs, was used as the benchmark geometry for this study. All the flutter analyses carried out in this paper are with frequency mistuning, but the possible consequences of mistuned mode shapes are briefly discussed at the end of this paper. Only the first family of modes (1F, first flap) is considered in this work. For the frequency mistuning analysis, the $1 \mathrm{~F}$ frequency is varied around the annulus but the $1 \mathrm{~F}$ mode shapes remain the same for all the blades. For the mode shape mistuning computations, an FE analysis of the whole assembly different mass blades is performed.

The results of this work clearly show the importance of mistuning on flutter. It also demonstrates that when using rig test data for aeroelastic validation of CFD codes, the amount mistuning present must be known.

Finally, it should be noted that the aim of this paper is the study of mistuning and not steady/unsteady validation of a CFD code and therefore minimal aerodynamic data are presented.

\section{NOMENCLATURE}

Aaero aerodynamic matrix

DF amplitude of mistuning

$f \quad$ blade natural frequency, $\mathrm{Hz}$

$f_{r} \quad$ reduced frequency, $f_{c} / V$

$i \quad i=\sqrt{-1}$

$K \quad$ stiffness matrix

$m \quad$ mass flow rate

$m_{\text {ref }} \quad$ normalized corrected mass flow rate

$M \quad$ mass matrix

$\omega \quad$ rotor angular velocity

$\Omega \quad$ rotor non-dimensional angular velocity

$\left(\omega 2 \pi r_{t} / a_{0}\right)$

ABM Alternating Blade Mistuning

AIC Aerodynamic Influence Coefficient

FMM Fundamental Mistuning Model

HOK High opertaing Line

\section{INTRODUCTION}

It is well known that mistuning can have significant effect on fan flutter. The vast majority of turbomachinery 
blade mistuning studies have been conducted using only the effects of structural coupling. However, aerodynamic coupling between blades can have significant effects on the stability and blade forced response amplitudes. The significance of the aerodynamic coupling effects has been shown in recent literature. For example, Kielb et al. [1, 2] and Miyakozawa [3] investigated the effects of aerodynamic coupling on both flutter and forced response using the single family of modes approach. The results suggested that the mistuning effects on flutter and forced response are dependent on the structural coupling levels. Campobasso et al. [4] conducted mathematical studies of flutter and forced response including the aerodynamic coupling effects assuming that the effect of the aerodynamic force is due to the blade motions of immediate neighbours. Sladojevic et al. [5] studied the correlation between the aerodynamic bladeto-blade coupling in mistuned bladed disk assemblies and the level of forced response. The analysis showed that the amplification factors were greatly increased due to aerodynamic coupling. He et al. [6] investigated the effects of aerodynamic coupling on both flutter and forced response problems using the tuned system modes to calculate the unsteady aerodynamic forces. For the case studied, aerodynamic coupling showed significant effects on the vibration amplitudes. All of these studies considered only blade frequency mistuning.

In the first part of this work, the effects of mistuning on flutter stability of a fan blade are studied by using a whole assembly non-linear time-accurate, viscous, model. Such computations contain many grid points and a range of frequencies, and hence are CPU intensive and cannot be performed routinely, but can provide a data base which can be used to examine simple mistuning models.

In the second phase of the work, mistuning is analyzed by using a low fidelity model. The simplified approach is based on a mistuned aeroelastic model which is reduced using the fundamental mistuning model (FMM) [1,7] and uses the aeroelastic influence coefficient matrix. The influence coefficients required for this algorithm are obtained from the time domain non-linear code by shaking one blade in the datum (tuned) frequency and mode. The analysis is done in three parts

1) Using a domain which contains the fan blade only. In this analysis, the damping is only a function of flow on the blade and mode shape and will be referred to as 'blade only' damping.

2) Using a domain which contains fan and intake. In this case, damping is function of intake acoustics, as well as flow and mode shape.

3) Using a domain which contains the entire low pressure (LP) compressor domain. This consists of fan, an intake, outlet guide vanes and engine-section stators. In such computation flutter stability is a function of both upstream and downstream reflections.

With this approach, one can establish whether mistuning modifies the 'blade only damping', upstream/downstream acoustics or both. Moreover, in the $1^{\text {st }}$ two stages of this work, the low and high fidelity models are compared extensively to establish the capabilities of the low fidelity model for flutter prediction. In the final part of this work the 'validated' aeroelastic fundamental mistuning model is used to define mistuned flutter boundaries for different levels/patterns of mistuning.

\section{THE AERODYNAMIC COMPUTATION METHOD}

The calculations in this paper were performed with the AU3D code written in Imperial College and developed over many years with support from Rolls-Royce [8]. The computations are performed using a three-dimensional, whole assembly, time-accurate, viscous approach. The unsteady flow cases are computed as Reynolds-averaged Navier-Stokes, with the basic assumption that the frequencies of interest are sufficiently far away from the frequencies of turbulent flow structures. The overall solution method is implicit, with second-order accuracy in space and time. The current computations use the one-equation Spalart-Allmaras turbulence model [9]. The parameters in Spalart-Allmaras have been adjusted on previous fans to get good agreement near the stability limit; the parameters are held constant in all the present work. The resulting CFD code has been used over the past 20 years for flows at off design conditions with a good degree of success $[10,11,12]$.

\section{AERODYNAMIC INFLUENCE COEFFICIENT METHOD}

The stability analysis of the bladed-disk is performed by an eigenvalue analysis of the aeroelastic problem [1, 13], which is defined by the following system

$$
M \ddot{\Phi}+C \dot{\Phi}+\left(K+A_{\text {aero }}\right) \Phi=0
$$

where K,C,M are the stiffness, damping and mass matrix of the bladed-disk (structure part) and $A_{\text {aero }}$ the complex aerodynamic matrix (fluid part). In this study the structural damping is not considered. The imaginary part of the eigenvalues defines the stability of the system. This approach has been successfully applied by several authors $[2,3,14]$.

The calculation of the aerodynamic matrix is based on aerodynamic influence coefficient method [7]. The idea is to have one harmonic excitation of the mode of interest $(1 \mathrm{~F}$ in here) of the blade 0 (excited blade) and measure (or calculate) the modal force on the neighboring blades. The influence coefficients are calculated as:

$$
\mathrm{A}_{\text {aero }}^{\mathrm{n}, 0}=\frac{\mathrm{F}_{\mathrm{n}}^{\mathrm{R}}+\mathrm{iF}_{\mathrm{n}}^{\mathrm{I}}}{\mathrm{q}_{0}^{\mathrm{R}}+\mathrm{iq}_{0}^{I}} \text { for the } n^{\text {th }} \text { blade }
$$

where, $F_{n}^{R}$ and $F_{n}^{I}$ are the real and imaginary parts of the aerodynamic force on the $\mathrm{n}^{\text {th }}$ blade due to the displacement of the blade $0 ; \mathrm{q}_{0}^{\mathrm{R}}$ and $\mathrm{q}_{0}^{\mathrm{I}}$ are the real and imaginary parts of the imposed modal displacement on blade 0 . The modal forces are obtained from $\mathrm{AU} 3 \mathrm{D}$ by shaking one blade (referred to as blade 0 ) in the $1 \mathrm{~F}$ mode at datum (tuned) frequency. The harmonic coefficients of the aerodynamic forces are obtained by an FFT of the AU3D transient simulation In the case of mistuning analysis the stiffness matrix $K$ contains the frequency mistuned pattern. In this part of the work, it is assumed that the disk is rigid and hence does not contribute to coupling of the blades. 


\section{TEST CASE AND STEADY FLOW}

A rig wide-chord fan blade, typical of modern civil designs, was used as the benchmark geometry for this study. The rig was used extensively to study flutter. There are 20 blades in the fan assembly. More details of test case can be found in [9]. Fig. 1 compares the calculated and measured stability boundary for this blade. The rotational speeds are nondimensionalised with respect to the sonic tip speed $(\Omega=$ $\left.\omega 2 \pi r_{t i p} / a\right)$ and the mass flows are non-dimensionalised with respect to the mass flow at the design point. The measured speedline is terminated to the left by the instability line and to the right is shown the normal working line. It should be noted that the instability can be stall/surge or flutter, with flutter occurring over a narrow range of fan rotational speed, referred to as 'flutter bite'. The stability line in Fig.1 is for flutter only, hence the AU3D steady speedlines can pass through the stability boundary (Flutter does not prevent CFD computations from going further up the constant speed characteristic). The flutter boundary has been obtained by performing around 5 flutter computations at each speed. The flutter point at each speed is defined as the point where the aero damping changes sign. The calculations, based on the known frequency and mode of vibration show instability due to flutter at somewhat higher mass flow rate than those measured. However, the trend is well predicted, notably the flutter bite (i.e., instability at increased throttle area) around $\Omega=0.89$.

The higher computed mass flow for instability is attributed to the presence of some mechanical damping and mistuning in the real fan which is wholly omitted in the present calculations. Partof the aims of this work is to see if the introduction of mistuning would bring the experimental and computed flutter boundaries closer and to determine the level of mistuning required for this purpose.

The dashed line (denoted by HWK) in Fig. 1 represents a high operating line which cuts through the flutter bite, and is used for understanding the effects of fan speed on flutter in this paper.

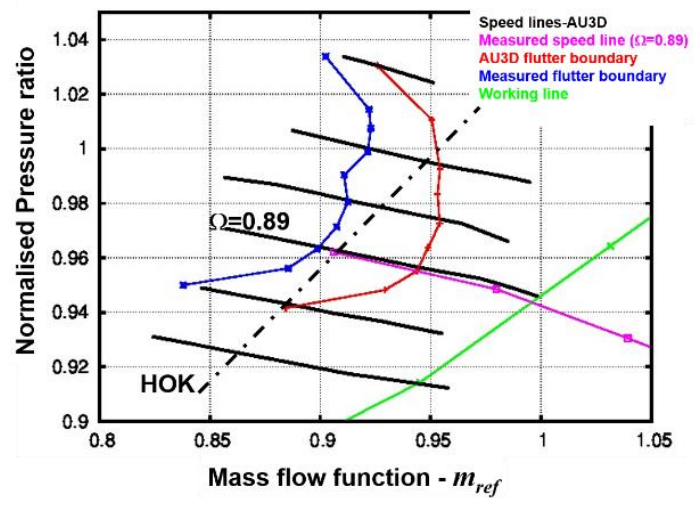

Fig 1. Performance plot for the model fan used in this investigation

In order to give the reader an idea of the flow at flutter condition $(\Omega=0.89$ ) for this blade some aero data is plotted in Fig 2. The contours of isentropic Mach number in the blade-to-blade surface at $90 \%$ span for mass flow of $\mathrm{m}=0.93$ when the relative velocity onto the rotor tip is close to sonic,
$\Omega=0.89$ is shown in Fig 2a. There is a well-developed supersonic region downstream of the leading edge, terminated by a shock at about $20 \%$ chord. Fig. 3 b shows the variation of static pressure along the blade at $90 \%$ height for different mass flows at $\Omega=0.89$. It is seen from this plot that for all the operating points considered at this speed, the shock is expelled. However, the shock moves forward and becomes stronger as the mass flow decreases. Moreover, it can be seen from this plot that the pressure is nearly uniform along the pressure surface and nearly uniform downstream of the shock on the suction surface. More details of steady flow can be found in [9].
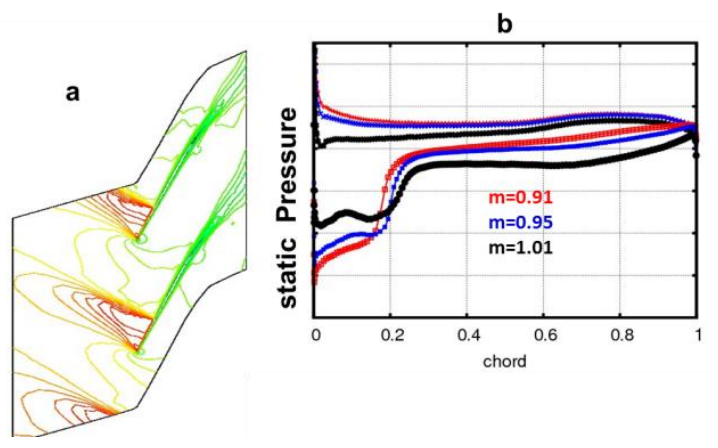

Fig 2: Typical view of steady flow at the speed. (a) Mach number contours at $90 \%$ height for $\mathrm{m}=0.93$; (b) Static pressure variations along the blade at $90 \%$ height

\section{MECHANICAL MODEL FOR FREQUECY MISTUNING STUDIES}

The mode shape and frequency used here were obtained from an FE package and were interpolated onto the CFD grid. The mode shapes remain fixed during computation. It is an underlying assumption that the mechanical mode is unaffected by the aerodynamic forces. Only the $1^{\text {st }}$ mode $(1 \mathrm{~F})$, shown in Fig. 3a, is considered at this stage.
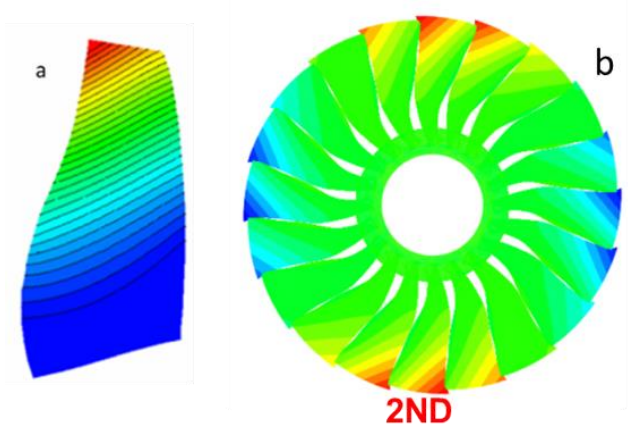

Fig. 3: (a) View of 1F mode shape for 1 blade, (b) Expaned 1F/2ND mode shape for whole assemblt

The usual approach in flutter computations is to expand the mode shape of Fig 3a for all the blades and Nodal Diameters (ND) of interest. Fig. 3b shows the 1F/2ND expanded mode. This method of expansion will be referred to as ND expansion of mode shape in the rest of this paper. Unsteady flutter computations begin from a steady state solution by exciting all the nodal diameters of interest. The amplitude of motion is large enough to be well outside the noise range, but small enough for the unsteady flow to be represented as 
linear; the imposed tip displacements are typically of the order of $0.3 \%$ of blade chord at the tip. The time history of displacement of each ND is tracked to establish whether that ND is stable or unstable. The stability of each mode is determined from the time history of the modal displacement. Fig 4a shows typical time history of displacement of a stable mode (decaying wave) and an unstable mode (amplifying wave). Aerodynamic damping is evaluated by fitting a line to the modal displacement amplitudes. The slope of the line on a $\log$ scale yields the logarithmic decrement (logdec) with a positive value corresponding to positive (stable) aerodynamic aero-damping and negative value corresponding to negative (unstable) aero-damping. Fig. 4b shows damping plotted against ND for mass flow of $\mathrm{m}=093$. It is seen from this plot that for this case $2 \mathrm{ND}, 3 \mathrm{ND}$ and $4 \mathrm{ND}$ are unstable in this example, whereas all the other NDs are stable.
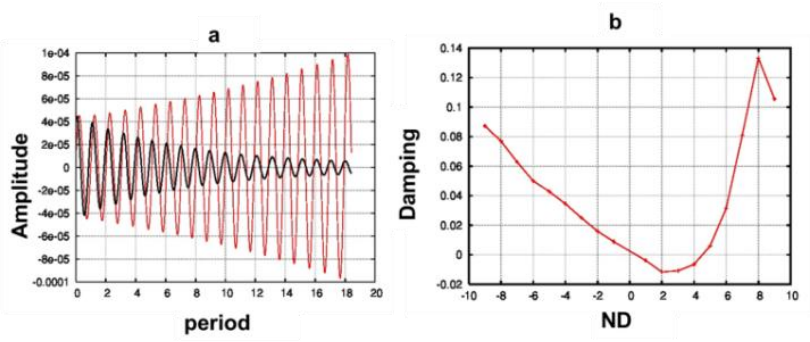

Fig 4: (a) Time history of displacement for a stable ND (black) and unstable ND (red); (b) Typical example of damping as a function of ND

The approach shown above is very effective for tuned systems as it allows the calculation of the aero damping for all the NDs in one computation. However, this approach cannot be used for frequency mistuned assemblies, as the expansion method used does not differentiate between blades (it can only distinguish between NDs), and hence one cannot assign different frequencies to different blades. Therefore, in the absence of mode shapes for the whole assembly, a new approach for expansion of mode shapes is required. In the approach taken here, it is assumed that the fan assembly displacement is made of 20 different $1 \mathrm{~F}$ modes, for which mode $\mathrm{N}$ corresponds to blade $\mathrm{N}$ having displacement while the displacement for all the other blades are zero in this mode. Modes 1, 5 and 13 are shown in Fig. 5. Therefore, with this approach, it is assumed that there is no structural coupling between blades and coupling is only through air. At time $\mathrm{T}=0.0$, only blade 1 is excited and the time history of all the blades are tracked. This method of mode shape expansion will be referred to as blade expansion in the rest of the paper.
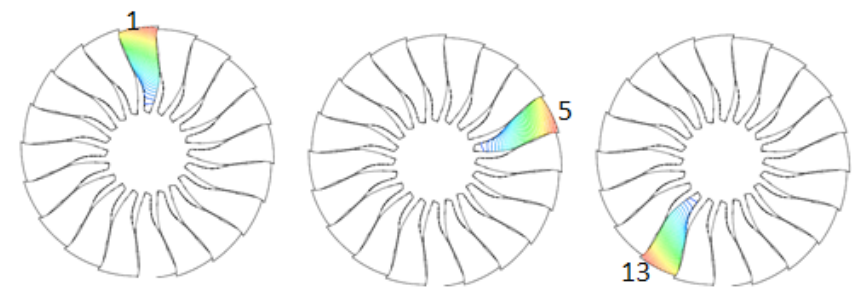

Fig 5: Alternative form of mode shape expansion; blades with no contours have zero displacement in the mode

\section{BLADE ONLY COMPUTATIONS}

Although the results in Fig 1 include an intake, outlet guide vanes and engine-section stators to allow comparison with measurement, initial calculations in this paper assume no reflections, corresponding to infinitely long intake and exhaust ducts. The removal of reflection is achieved in the calculations by coarsening the computation mesh upstream and downstream of the rotor. This type of computations is much faster than the whole LP analysis and allow the study of many parameters. In this model, aero damping is only a function of the flow, mode shape and frequency of the blade and is referred to 'blade only' damping [15]. The effects of mistuning on reflection is studied later in this paper.

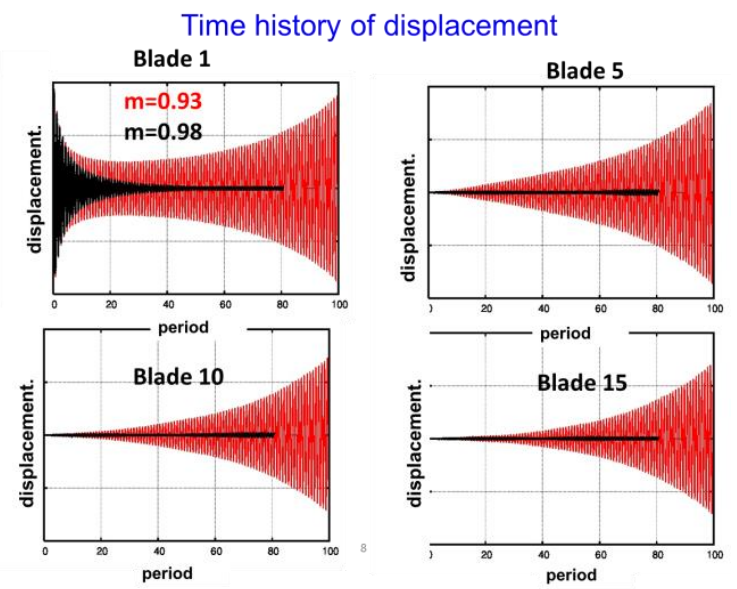

Fig 6: Time history of displacement for 4 blades around the annulus, $m_{\text {ref }}=0.93$ unstable; $m_{\text {ref }}=0.98$ stable

Before proceeding to mistuned computations, the blade expansion of the mode shape was checked. Fig 6 shows the time history of displacement for 4 blades around the assembly for a stable point $\left(m_{r e f}=0.98\right)$ and an unstable point ( $m_{\text {ref }}=0.93$ ). It should be noted that at $\mathrm{T}=0.0$ only blade 1 is excited.

It can be seen from Fig 6 that initially (up to 10 periods of vibration) the time history of displacement for both mass flows is similar. However, for $m_{\text {ref }}=0.93$ the initial disturbance of blade 1 is able to travel around the annulus and excite other blades and cause flutter, whereas for $m_{\text {ref }}$ $=0.98$ the initial excitation is unable to cause vibration of other blades. This can be clearly seen from Fig 7 which shows displacement plotted as a function of blade number for 5 instants of time during the motion.
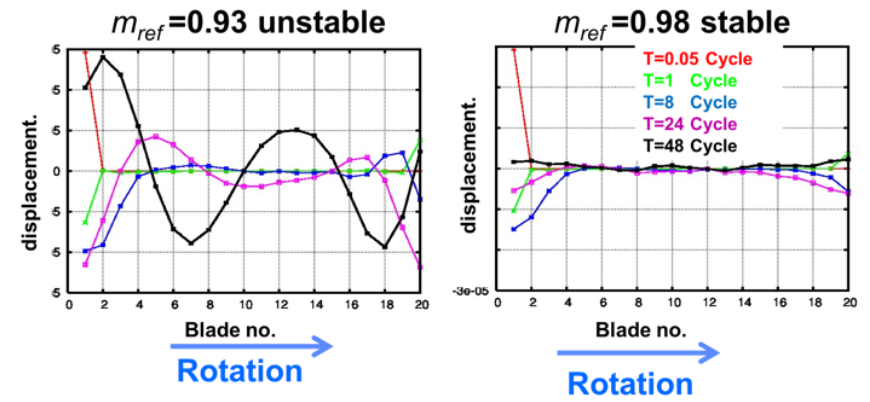

Fig 7: Time history of displacement as a function of blade number 
It is seen from this plot that at $\mathrm{T}=0.05$ cycle (red curve) and $\mathrm{T}=1$ cycle (green curve) the displacement is localised (around blade 1) and does not differ between the two mass flows. At $\mathrm{T}=8$ cycles (blue curve) it is still hard to distinguish between the two mass flows cases, although for $m_{\text {ref }}=0.93$ more blades $(19,18$ and 17) show vibration. At $\mathrm{T}=24$ cycle (pink curve), the characteristic of vibration is totally different between the two mass flow cases and all the blades for $m_{\text {ref }}=0.93$ show response. At $\mathrm{T}=48$ cycles (black curve), a clear 2ND pattern is developed for $m_{\text {ref }}$ $=0.93$, whereas for $m_{r e f}=0.98$ all the vibration has died out. The results clearly shows that aero coupling is sufficient to determine the least stable ND. Fig 8 shows the comparison of damping between the ND and blade expanded mode shapes. For the ND expanded mode shapes, the damping curve is for a 2ND pattern. For the blade expanded mode shape computations, the unstable points at $m_{\text {ref }}=0.93$ and $m_{\text {ref }}=0.91$ converge to a $2 \mathrm{ND}$ pattern. It is seen from this figure that, as expected, the ND expanded mode shape and blade expanded mode shape give the same results. Also shown in Fig 8 are the aero-damping obtained by using the low fidelity AIC method. It is seen from this plot that, with the exception of a slight difference at $m_{\text {ref }}=0.91$ (where the flow is very close to stall), the AIC method gives comparable damping values to AU3D and furthermore predicts the same mass flow at flutter. Therefore, it can be concluded that for the tuned system, AIC can be used for flutter prediction of this blade.

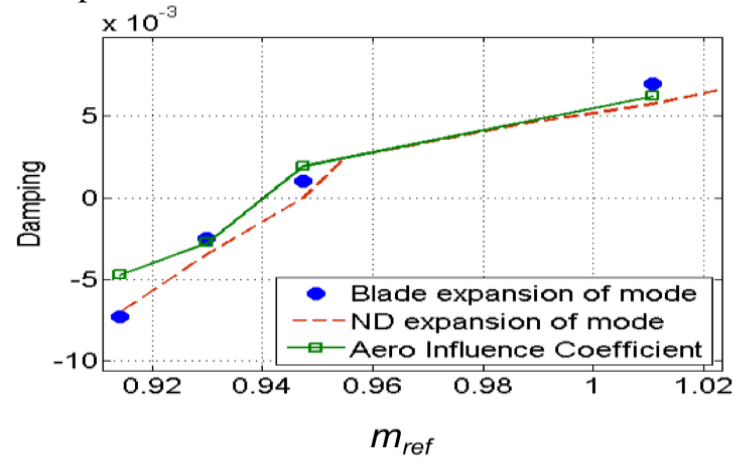

Fig 8: Comparison of damping between ND, blade expend mode shape and aero influence coefficient

The frequency mistuned pattern used for this part of study together with its harmonics is shown in Fig 9. The pattern has been obtained using a random number generator. The mode shape of blades is assumed to remain the same and is not affected by mistuning. The amplitude of mistuning (DF) which is defined as:

$$
\mathrm{DF}=0.5^{*}\left(\mathrm{~F}_{\max }-\mathrm{F}_{\min }\right) / \mathrm{F}_{\mathrm{ave}}
$$

where $F_{\max }, F_{\min }$ and $F_{\text {ave }}$ are maximum, minimum and average frequencies respectively. For the case shown in Fig 9 the amplitude of mistuning is $3 \%$.
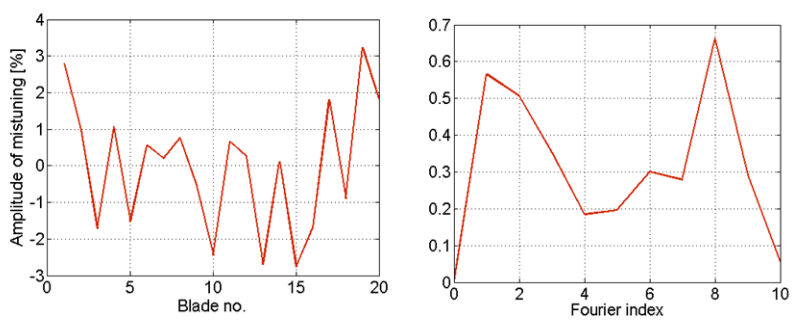

Fig 9: Frequency mistuning pattern used in this study

Initially, the computations were performed for unstable mass flow of $m_{\text {ref }}=0.93$. Fig 10 shows the time history of displacement for 4 blades around the assembly for the tuned and the mistuned fan assembly. It is seen that the addition of mistuning pattern of Fig 9 has made the fan assembly stable at $m_{r e f}=0.93$. On comparison with Fig 6, it is seen that the time history of mistuned assembly at $m_{\text {ref }}=0.93$ is very similar to that of the tuned system at $m_{\text {ref }}=0.98$. This suggests that the introduction of mistuning stops the initial disturbance from propagating around the circumference at this mass flow.
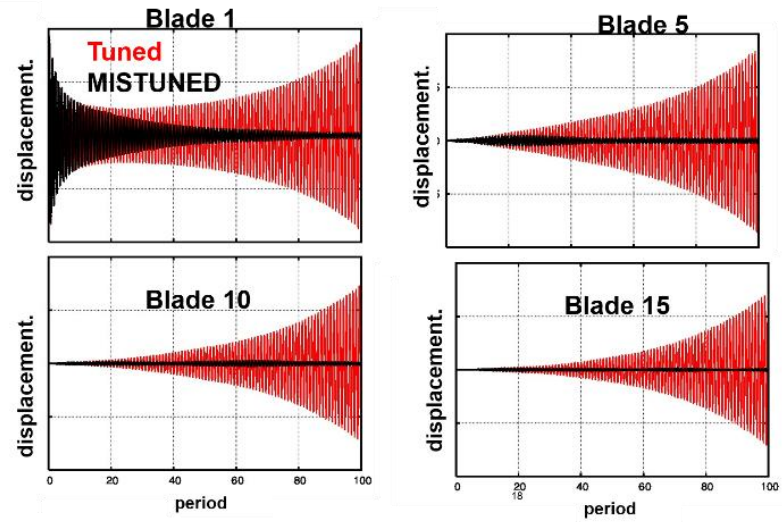

Fig 10: Time history of displacement for 4 blades around the annulus at $m_{\text {ref }}=0.93$

Fig 11 which shows the FFT of displacement (for $m_{\text {ref }}=0.93$ ) around the circumference after 100 cycles for the tuned and mistuned systems (denoted by MS*5). It is seen from this plot that for the tuned system the 2ND is the only mode present, whereas for the mistuned system, one can observe the presence of other NDs in response (1-5). It should be noted that the mistuned plot curve in Fig $\mathbf{1 1}$ has been multiplied by 5 so that it is visible in the plot.

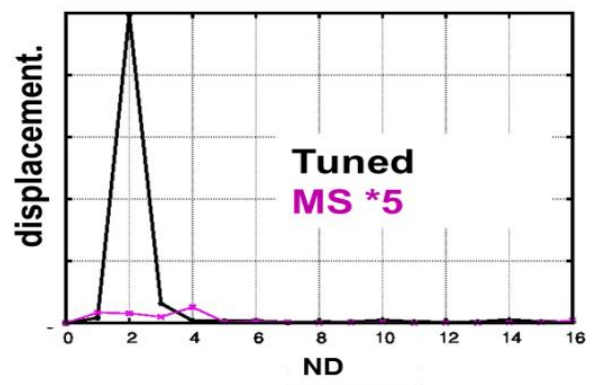

Fig 11: Fourier transform of displacement along the circumference at $m_{\text {ref }}=0.93$ 
Fig 12 shows the frequency content of displacement for blades 10 for the mistuned computations at $m_{\text {ref }}=0.93$. The $\mathrm{x}$-axis is normalised by the datum (tuned) frequency. It is seen from this plot that the blade responds in a range of frequencies around the average frequency and therefore, the time histories (Fig 10) for mistuned systems are non-linear and hence the concept of damping is not valid anymore. Moreover, due to interaction between blades, low frequency components are present in the response. The presence of these low frequency components is the main reason that non-linear time domain mistuning computations are very time consuming as these frequencies can differ from blade frequency by orders of magnitude.

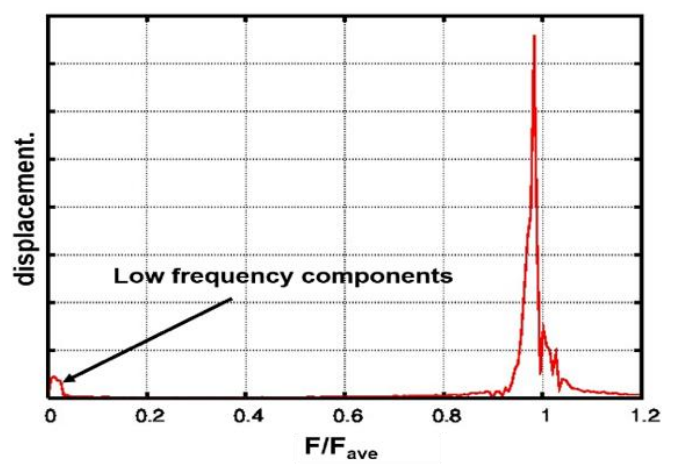

Fig 12: Fourier transform of time history displacement for two blade for mistuned case.

Fig. 13, shows the computed aero damping plotted against mass flow for a tuned system and the frequency mistuned pattern of Fig 9. Both non-linear time domain and AIC method results for mistuning are included in this plot.

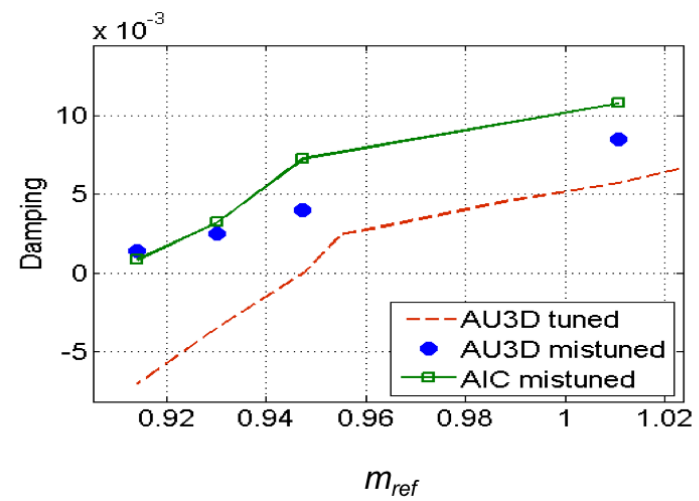

Fig 13: Comparison of aero damping for tuned and mistuned assembly

It is seen from Fig 13 that the non-linear model and the AIC method compare well for the mistuned case, however, the magnitude of damping for the stable mass flow cases $\left(m_{\text {ref }}\right.$ $>0.93$ ) is bigger with the AIC method than with AU3D. This discrepancy can be explained by the fact that it is difficult to accurately quantify damping for stable cases with (nonlinear) AU3D with mistuning. The results show that for the tuned system as the mass flow decreases, there is a sharp drop in aero damping and the blade flutters at mass flows below 0.95 . However, for the pattern and level of mistuning used in these computations the blade remains stable at all the computed mass flows and the fan blade stalls before it flutters. It should be noted that, the concept of 'damping' is only valid when the time histories are linear. As shown above, the time history for the mistuned cases is non-linear and hence obtaining a damping from a non-linear curve is quite approximate. The approximation used here is described in detail at the end of this section.

The effect of amplitude of mistuning on flutter was considered next. Fig 14 shows the aero damping of the blade for $m_{\text {ref }}=0.91$ and $m_{\text {ref }}=0.93$ plotted against the amplitude of mistuning (equation 3). It should be noted that the same pattern (Fig 9) of frequency mistuning is used in all the computations shown in Fig 14 and only the amplitude of mistuning (DF) is changed. The tuned system is shown as $0 \%$ mistuning level. It is apparent from this plot that for the pattern of mistuning used here, the AIC and AU3D give very similar results. Moreover, it is seen from this plot that amplitude of mistuning has a significant effect on the aero damping of the blade. For the particular pattern used here, at $m_{\text {ref }}=0.93$ a $1.2 \%$ amplitude is required for the blade to become stable. It is also seen from this plot that, for the particular pattern of mistuning used here, the aero damping of the blade asymptotes as the amount of mistuning is increased. Therefore, increasing mistuning (above 3\%) will not increase the flutter margin of this fan blade any further.

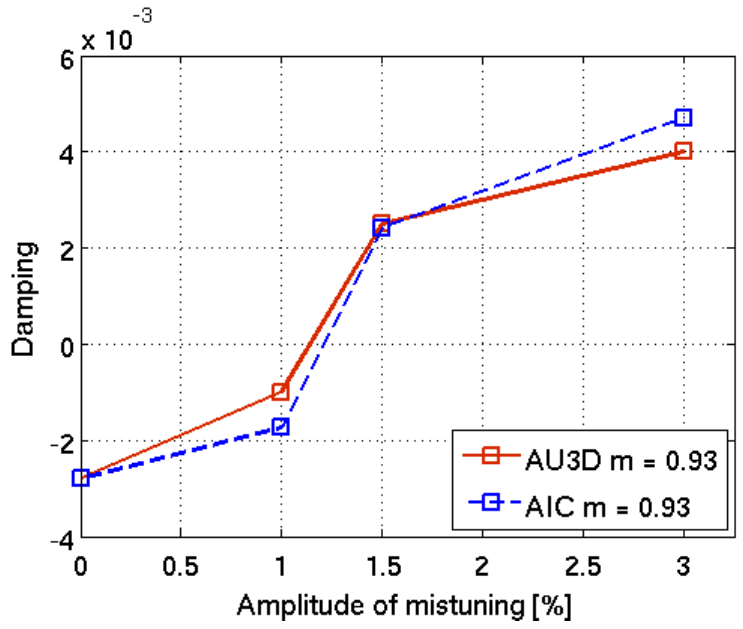

Fig 14: Aerodamping as a function of amplitude of mistuning for the random pattern at $m_{\text {ref }}=0.93$

Fig 15 shows the frequency content of response for $m_{r e f}$ $=0.93$ at $1 \%$ mistuning and at $3 \%$ mistuning. The curve for $3 \%$ mistuning (denoted by $3 \%$ mistuning*10) has been multiplied by 10 so that it is visible in this plot. It can be seen from this plot that for the stable case (3\% mistuning) the blade responds in a range of frequencies, whereas as at the unstable situation (1\%) the blade responds at (nearly) a single frequency. Therefore, mistuning improves flutter stability by not allowing the system to respond in a single frequency. Therefore, for an unstable system it is possible to obtain a value for damping as all the blades respond (nearly) at the same frequency. However, for a stable system the value of damping is somewhat arbitrary as there are many frequencies present in the response of each blade and the concept of damping does not really exist. For the stable points shown in this paper, the value of damping stated (or 
shown in a plot) is obtained from average damping of all the blades over many time intervals. Ideally, one needs to obtain the amplitude of limit cycle for these cases but the CPU requirement to complete such a task is outside the scope of practical engineering. However, in practice, the value of damping for a stable assembly is not really significant. It is only important to know if the blade is stable or unstable and, if unstable, what is the value of negative damping. Therefore, in plots such as Fig 13 and Fig 14 the most important features are:

1) The location when the damping curve crosses the $x$ axis(zero damping), which defines the flutter boundary,

2) The values of damping when they are negative, which defines the amount of damping required to make the blade stable.

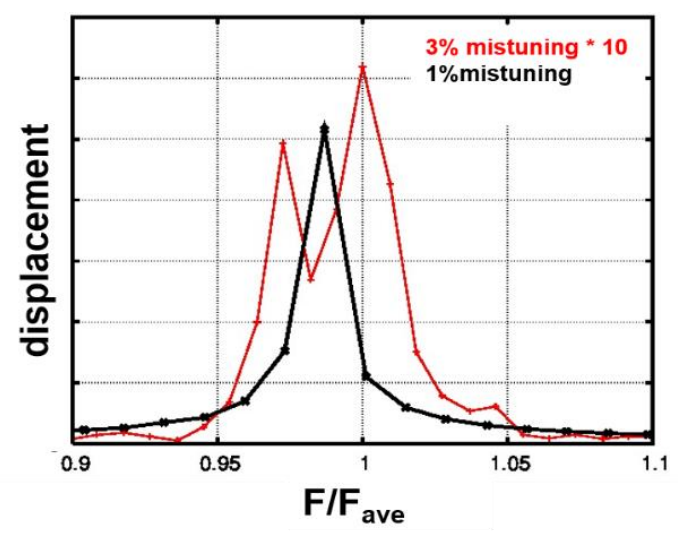

Fig 15: Frequency components of displacement for a stable and an unstable point using AU3D

\section{SOME INSIGHT INTO BEHAVIOR DURING MISTUNING}

Although, non-linear computations with AU3D are very expensive, they can provide an insight into the unsteady aerodynamic flow and aeroelastic behaviour of mistuned blades. In this section some of the feature observed from the non-linear analyses is presented. In these set of computations, the computations start with exciting the assembly in a 2ND pattern (see Fig 16 red curve).

\section{Stable case}

In this set of the computations a $3 \%$ mistuning at $m_{\text {ref }}=0.93$ is considered. For a tuned case, the initial pattern would remain unchanged but its amplitude would increase at this (unstable) mass flow. It is seen from Fig $\mathbf{1 6}$ that this is not the situation for a mistuned case. After only 15 cycles of vibration, the $2 \mathrm{ND}$ pattern has broken down to many circumferential modes and from the Fourier harmonic plot, it is seen that $1 \mathrm{ND}, 3 \mathrm{ND}$ and $4 \mathrm{ND}$ are also present in the assembly response. After, 30 cycles, the 3ND shows the biggest response levels, and after 60 cycles, the circumferential modes from 1 to 7 are present in the response. This plot clearly shows that, although the system is excited in such a way that, the initial energy is only in a 2ND mode, the introduction of mistuning distributes this energy over many circumferential modes. Moreover, as can be seen from Fig 3b most of the other modes are stable and hence dissipate this energy resulting in a stable system. This observation is similar to the one shown in [16] for compressor blades.
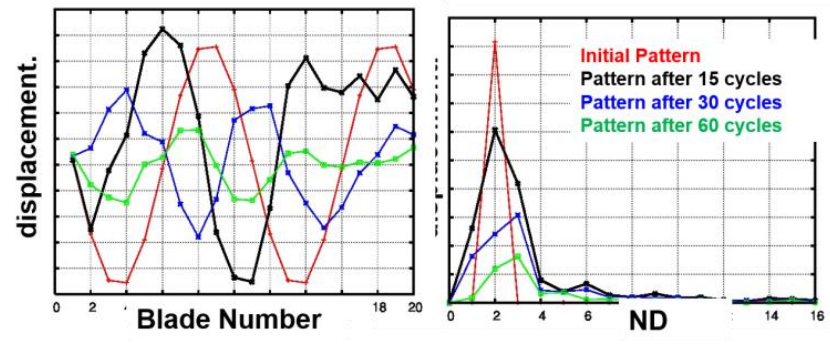

Fig 16: Time history of displacement as a function of blade number and the Fourier harmonic for the stable case $\mathrm{m}=0.93$ and $6 \%$ mistuning - Stable

\section{Unstable case}

This set of computations corresponds to a $1 \%$ mistuning at $m_{\text {ref }}=0.93$, for which the amount of mistuning is not sufficient so stabilise the assembly. The variations of displacement around the circumference after 0 and 40 cycles vibration together with their Fourier components for this case are shown in Fig 17. It is seen from this figure that, although mistuning has managed to distribute the initial 2ND energy over many circumferential modes, the large proportion of energy still remains within a 2ND pattern which is unstable. Therefore, in this case, mistuning is not able to terminate flutter and can only decrease the amplitude of (negative) damping.
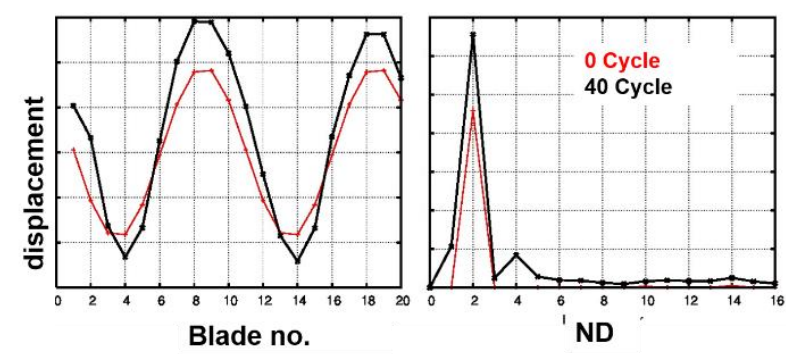

Fig 17: Time history of displacement as a function of blade number and the Fourier harmonic for the stable case $m_{\text {ref }}=0.93$ and $1 \%$ mistuning -Unstable

\section{ANLAYSIS WITH DIFFERENT PATTERNS OF MISTUNING}

In this section the effects of pattern of mistuning on stability is analysed. Alternate blade mistuning is, generally, considered as the best way to stabilize or increase flutter margin of bladed-disk [13]. In order to verify this statement, different mistuned patterns are tested. The patterns are in the form:

$$
P=\sin \left(\frac{k}{n_{\text {blade }}} j 2 \pi\right)
$$

where $\mathrm{k}$ is the Fourier index, $n_{\text {blade }}$ is the number of blade in the annulus and $\mathrm{j}$ is the blade index. For a 20 bladed disk alternate mistuning corresponds to $\mathrm{k}=10$. The computations in this section are at $m_{\text {ref }}=0.91$. Fig 18 shows the comparison of AU3D and AIC results for $\mathrm{k}=2$ (cos $(2 * \theta))$ and $\mathrm{k}=10$ (alternate mistuning). It can be observed 
that, both methods predict the same trend, however the AIC method predicts higher values of damping for $\mathrm{k}=10$ (alternate mistuning)and DF $>2 \%$. This discrepancy only affects the stable region. Again, it is difficult to know if it is, due to a lack of accuracy of AIC method or an inappropriate method of computing damping in AU3D for stable modes. However, these discrepancies do not affect the findings of this paper as they occur when the blade is stable, and hence will not change the flutter boundary.

It is also observed from this plot that (surprisingly) $\mathrm{k}=2$ mistuned pattern is the most efficient way to avoid flutter in this particular case (which is a 2ND flutter for 20 bladed disk). Kielb et al [13] have also shown that wave patterns mistuning can stabilize the flutter of bladed-disks. However, in the case considered in [13], the result showed that for a 5ND flutter of 35 bladed disk alternate mistuning is the best way of controlling flutter, which is in contradiction with the case studied here.

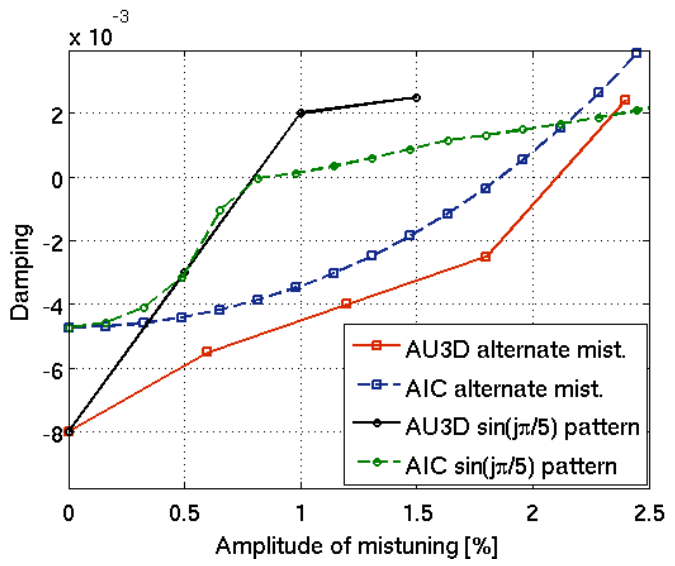

Fig 18: Aero damping as a function of amplitude of mistuning for different patterns of mistuning at mass flow of $m_{\text {ref }}=0.91$

The results from AIC computation are drawn as colour map in Fig 19. This type of plot is very useful for establishing the best pattern of mistuning for a particular case as the border between green and red defines the flutter boundary. Fig 19 clearly shows that in this case increasing k (apart from $\mathrm{k}=10$ ) reduces the effects of mistuning on flutter.

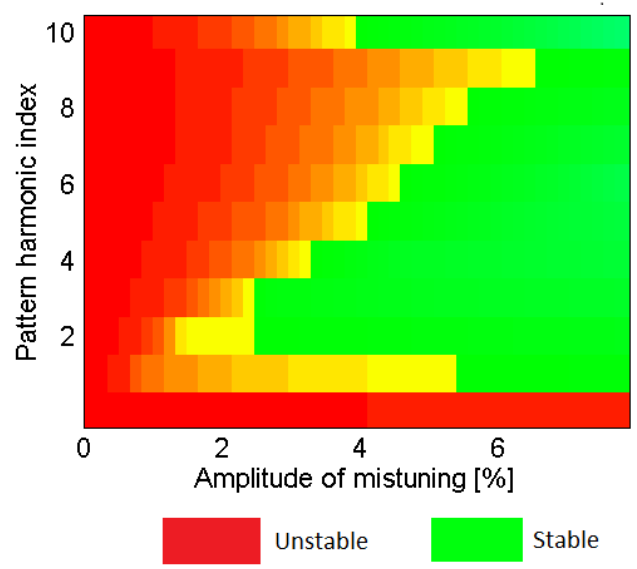

Fig 19: Stability map as a function of amplitude of mistuning and harmonic pattern mistuning at mass flow of $m_{\text {ref }}=0.91$

\section{COMPUTATIONS WITH INTAKE}

It was shown in $[15,17]$ that the intake acoustics play a very important part in fan flutter. This is illustrated in Fig 20 (taken from [15]) which shows the comparison of damping as a function of tip speed for a case with and without intake. The results of [15] also showed that the introduction of intake will shift the least stable mode to a 3ND pattern. In this section the combined effects of mistuning and intake on fan flutter is studied. The mistuning pattern used for validation of AIC is the one shown in Fig 9. Computations are performed at a tip Mach number of $\Omega=0.89$ which is the most unstable case in Fig. 20.

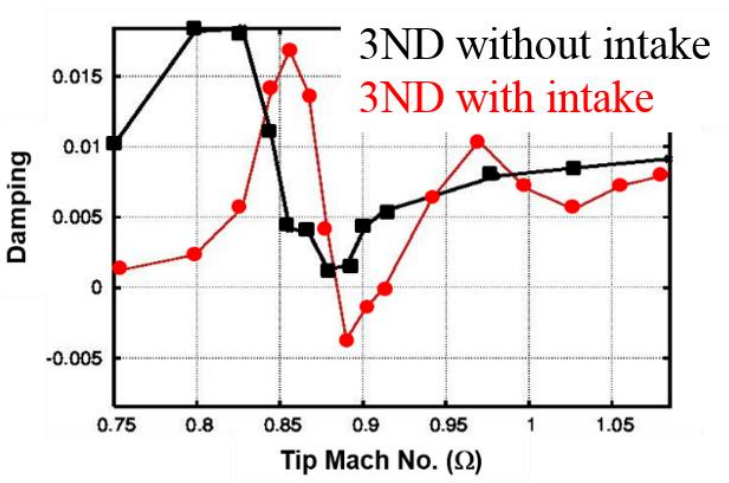

Fig 20: Computed damping on a constant operating line against non-dimensional tip speed $\Omega$

It should be noted that the results of Fig $\mathbf{2 0}$ have been obtained using a mode shape expansion strategy. Therefore, the computations are performed for the tuned and mistuned case using a blade expansion of the mode shape. Fig 21 shows the instantaneous displacement around the circumference for the tuned and mistuned cases, together with their Fourier harmonics. Again, the mistuned curve is multiplied by 5 so that it is visible in the plot.
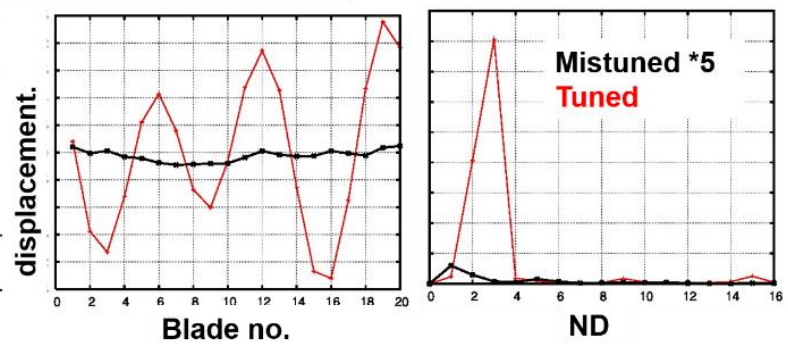

Fig 21: Time history of displacement as a function of blade number and the Fourier content for case with intake at Mach number of 0.89

It is seen that the inclusion of the intake moves the least stable mode from a 2 ND pattern to a 3 ND ptteren, which is in line with the results of [17] and mesaured data. Moreover, it is seen from this plot, that this pattern and amplitude of mistuning will stabilize the blade. In the next phase of this work, computaions with aerodynamic influence coefficient method was considered. The influence coefficients required for AIC analysis were obtained by performing whole annulus non-linear computations with intake and shaking one of the blades in a $1 \mathrm{~F}$ mode at the tuned frequency. Fig 
$\mathbf{2 2}$ gives the comparison of damping between AU3D results and the AIC method for the tuned and mistuned cases. It is seen from this figure that reasonable agreement exists between the two methodologies. Following the same idea presented in the previous section the effects of different wave patterns on mistuning was studied by using AIC method. Fig 23 shows that in this case alternating blade mistuning $(\mathrm{k}=10)$ is almost as efficient as $\mathrm{k}=1$ or $\mathrm{k}=2$ mistuning pattern for stabilising the bladed disk. Even if alternating blade mistuning is not the optimal pattern for stabilizing a specific configuration it is a robust pattern for stabilizing any configuration. These results agree with previous works $[3,16]$ which recommend the use of intentional alternating blade mistuning.

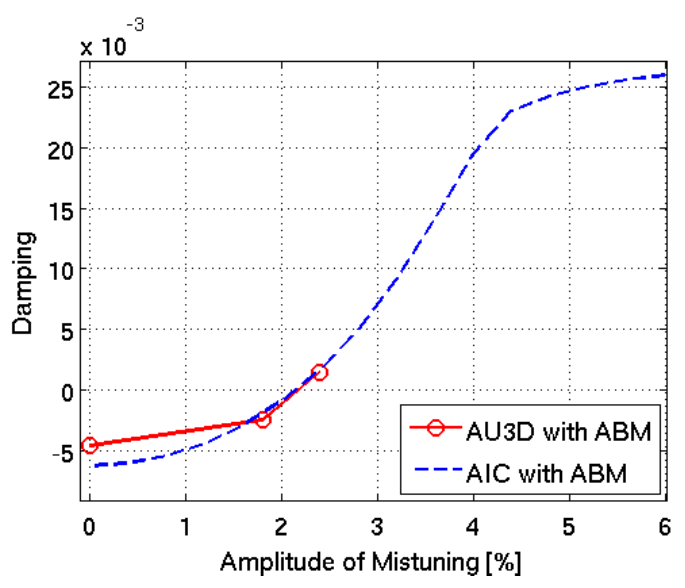

Fig22: Comparion of results between AU3D and AIC method at tip Mach number of 0.89

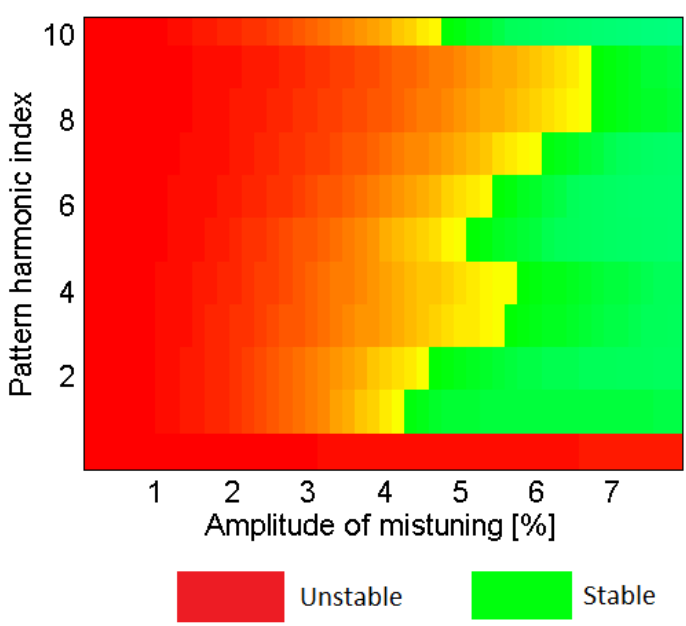

Fig 23: Stability map as a function of amplitude of mistuning and harmonic pattern mistuning at mass flows of $\mathrm{m}=0.91$ with intake

\section{COMPUTATION OF WHOLE LP}

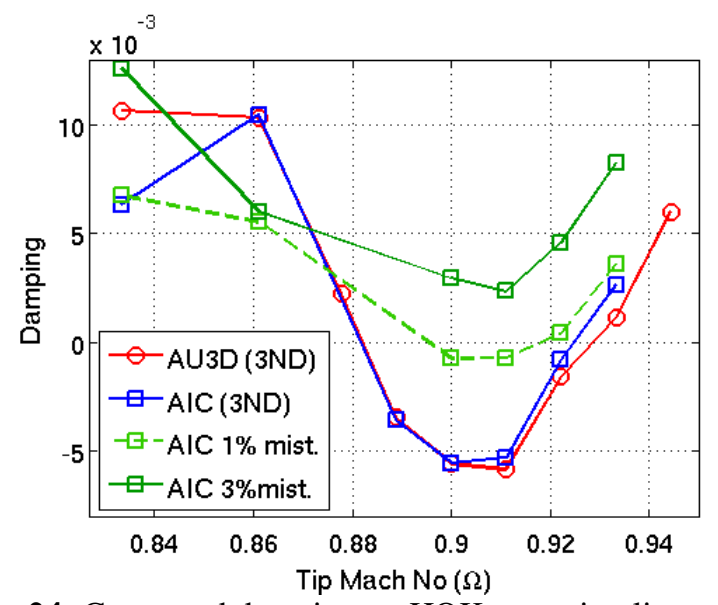

Fig 24: Computed damping on HOK operating line against non-dimensional tip speed $\Omega$ with tuned and mistuned blades

In this section the results for the whole LP are presented. All the mistuning results in this section have been obtained by using AIC method. Once again, the required influence coefficients were obtained by using a whole assembly analysis of the entire LP and shaking one the blades in $1 \mathrm{~F}$ mode and its datum frequency. Fig $\mathbf{2 4}$ shows the variations of damping plotted against aero speed for the operating line HOK shown in Fig 1. There are 3 plots in this curve which correspond to tuned analysis with non-linear code, tuned analysis with AIC method and the mistuned analysis with AIC method. The mistuned analysis was performed with the same pattern as shown in Fig 9. AU3D and AIC simulations agree well for the tuned case. It is seem from this plot that, a $3 \%$ mistuning is sufficient to stabilize the bladed-disk at this operating line.

\section{COMPUTATIONS WITH MISTUNED MODES}

The results in previous section were obtained by considering a frequency mistuning. In this section, the real mode shape mistuning is studied. In order, to establish the effect of mode shape mistuning an FE analysis of the whole assembly with mistuning was performed. Mistuning was obtained by adding a lumped mass to the mesh at the tip of each blade as shown in Fig 25a. The values of the masses are chosen in such a way to create the required mistuned pattern. 


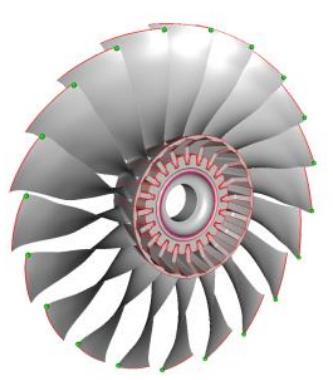

a
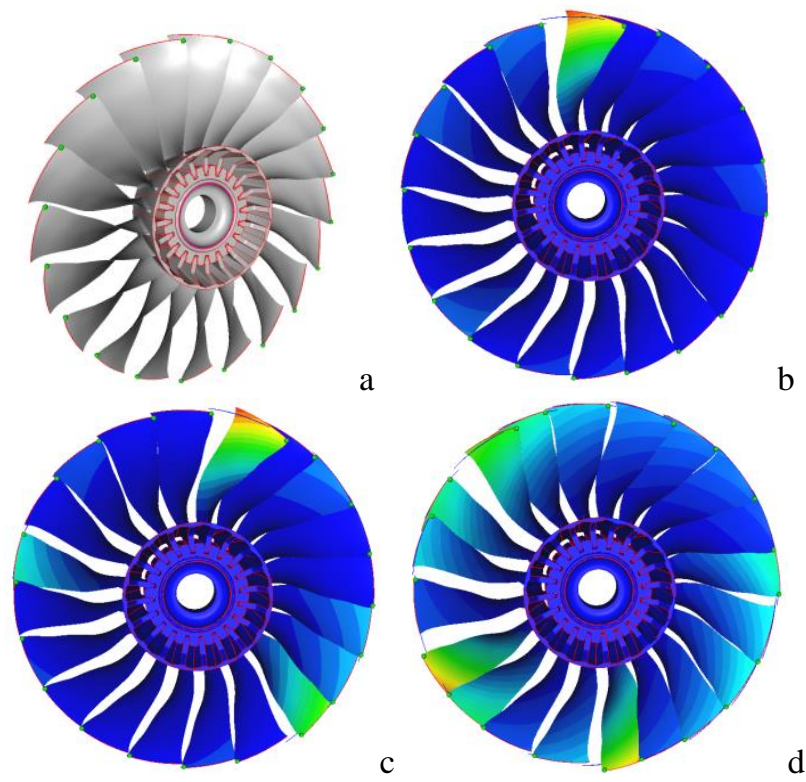

Fig 25: Mistuned blade disk FE model (a) with different mode shapes (b,c,d). Green circles show the location of added masses in (a)

Examples of mistuned mode shapes for the first 1F mode family are shown on Fig25b, c and d. Fig 25b shows a mistuned mode shape with localization, for which only one blade responds. This kind of mode shape can be modelled by single blade mode shape and frequency mistuning as it was done in this study. Mode shapes illustrated in Fig25c and $\mathbf{d}$ have coupling effects through the disk. It will be necessary to input the whole annulus modeshape in AU3D to take into account this coupling. It will be the purpose of a future work, to validate AIC method against AU3D results obtained with mistuned mode shapes.

\section{CONCLUSIONS AND FUTURE WORK}

The results presented in this paper clearly show that mistuning has a substantial effect on flutter stability of the fan blade and can increase the flutter margin of a fan blade significantly. Computed CFD results show that using a $3 \%$ random mistuning pattern will bring the computed and measured flutter boundary closer together. The result here, also show that the flutter boundary is very sensitive to the amount of mistuning present. Therefore, it is not surprising that different engines (rigs) may have different flutter boundaries.

In order to accelerate the analysis of mistuning effect the aerodynamic influence coefficient method has been implemented and validated against full nonlinear aerodynamic simulation in AU3D. The results show that the method is valid and can be used to study different mistuning pattern and amplitude of mistuning.

In this study the mistuning was applied using blade frequency mistuning. Future work will focus on effect of mistuned mode shapes on aerodynamic response and validity of AIC method in that case.

\section{Acknowledgements}

The authors thank Rolls-Royce plc for both sponsoring this work and allowing its publication. The first author also wishes to acknowledge support from UK TSB under the SILOET project. They gratefully acknowledge the contribution of their colleagues at Rolls-Royce plc: J. Green, R. Brooks, M. Wilson, K. Johal and S. Stapelfeldt.

\section{REFERENCES}

[1] Kielb, R. E., Feiner, D. M., Griffn, J. H., and Miyakozawa, T., 2004. , "Flutter of mistuned bladed disks and blisks with aerodynamic and FMM structural coupling". Proceedings of the ASME Turbo Expo 2004, Vienna, Austria, June 14-17, 2004, 6, pp. 573-579

[2] Kielb, R. E., Feiner, D. M., Griffn, J. H., and Miyakozawa, T., March,2005. "Probabilistic analysis of mistuned bladed disks and blisks with aerodynamic and FMM structural coupling". 9th National Turbine Engine HCF Conference.

[3] Miyakozawa, T, 2008, "Flutter and forced response of turbomachinery with frequency mistuning and aerodynamic asymmetry", $\mathrm{PhD}$ thesis, Department of Mechanical Engineering and Materials Science Duke University

[4] Campobasso, M., and Giles, M., 2000. "Flutter and forced response of mistuned turbomachinery", Oxford University Computing Laboratory Report NA 00/20.

[5] Sladojevic, J., Petrov, E., Sayma, A., Imregun, M., and Green, J., 2005. "Investigation of the influence of aerodynamic coupling on response levels of mistuned bladed discs with weak structural coupling". Proceedings of the ASME Turbo Expo, 4, pp. 543 - 551.

[6] He, Z., Epureanu, B. I., and Pierre, C., 2007. "Fluidstructural coupling effects on the dynamics of mistuned bladed disks". AIAA Journal, 45(3), pp. 552 - 561

[7] Hsu, K., and Hoyniak D., "A Fast Influence Coefficient Method for Linearized Flutter and Forced-Response Analysis", 49th AIAA Aerospace Sciences Meeting Including the New Horizons Forum and Aerospace Exposition, Orlando, FL, 2011.

[8] Sayma, A. I., Vahdati, M., Sbardella, L., and Imregun, M., 2000, "Modeling of Three-Dimensional Viscous Compressible Turbomachinery Flows Using Unstructured Hybrid Grids," AIAA J., 38(6), pp. 945-954

[9] Spalart, P. R., and Allmaras, S. R., 1992, "A OneEquation Turbulence Model for Aerodynamic Flows," AIAA Paper No. 92-0439 
[10] Vahdati, M., and Cumpsty, N. A., 2012, "Mechanisms for Wide-Chord Fan Blade Flutter," 13th International Symposium on Unsteady Aerodynamics, Aeroacoustics and Aeroelasticity of Turbomachines, University of Tokyo, Japan, Sept. 11-14, Paper No. ISUAAAT 13-I-5.

[11] Choi, M., Smith, N.H.S and Vahdati. M, 2013, "Validation of numerical simulation for rotating stall in a transonic fan”, Journal of turbomachinery 135 (2), 021004

[12] J Dodds, J., Vahdati, M., 2015, "Rotating Stall Observations in a High Speed Compressor-Part II: Numerical Study", Journal of Turbomachinery 137 (5), 051003

[13] Temis, Y.M. and Salles L., "Efficient CSM/CFD method for aeroelasticity stability analysis of turbomachine blades" ISROMAC 14, Honolulu, 2012

[14] Nipkau, J., Kühhorn A., and Beirow B., "Modal and Aeroelastic Analysis of a Compressor Blisk Considering Mistuning", ASME Turbo Expo, Vancouver 2011.

[15] Vahdati, M, Smith, N.H.S, Zhao, F., 2015, "Influence of Intake on Fan Blade Flutter", Journal of Turbomachinery 137 (8), 081002

[16] Kielb, R. E., Hall, K. C., Hong, E., and Pai, S. S., 2006, "Probabilistic Flutter Analysis of a Mistuned Bladed Disks," ASME Turbo Expo Paper No. GT2006-90847, Barcelona, Spain, May 8-11, 2006

[17] Vahdati, M., Simpson, G., Imregun, M., 2011, "Mechanisms for wide-chord fan blade flutter", Journal of Turbomachinery 133(4), 041029

[18] Vahdati M, Cumpsty N, Aeroelastic Instability in Transonic Fans, Journal of Engineering for Gas Turbines and Power, Vol 138, 022604, Feb 2016 